
allemande

48-1 | 2016

Les expériences coloniales allemandes : échanges, transferts, circulations

\title{
La Galicie autrichienne : « colonie du bois » de l'Empire allemand ? (1890-1914)
}

Jawad Daheur

\section{(2) OpenEdition}

\section{Journals}

Édition électronique

URL : https://journals.openedition.org/allemagne/364

DOI : 10.4000 /allemagne. 364

ISSN : 2605-7913

Éditeur

Société d'études allemandes

Édition imprimée

Date de publication : 15 juin 2016

Pagination : 25-42

ISSN : 0035-0974

Référence électronique

Jawad Daheur, «La Galicie autrichienne : « colonie du bois » de l'Empire allemand ? (1890-1914)», Revue d'Allemagne et des pays de langue allemande [En ligne], 48-1 | 2016, mis en ligne le 13 décembre 2017, consulté le 18 mai 2021. URL : http://journals.openedition.org/allemagne/364 ; DOI : https:// doi.org/10.4000/allemagne.364 


\section{La Galicie autrichienne: «colonie du bois » de l’Empire allemand? (1890-1914)}

\section{- Jawad Daheur*}

Dans un article de 1974, Woodruff D. Smith identifiait le "colonialisme économique» comme l'une des deux formes de la pensée coloniale allemande au XIX ${ }^{\mathrm{e}}$ siècle, aux côtés d'un «colonialisme émigrationniste» favorable à l'établissement de colonies de peuplement ${ }^{(1)}$. Élément essentiel de la Weltpolitik de Guillaume II à partir de 1890, l'approche «économique» reposait sur l'idée d'une division du monde en vastes zones commerciales en compétition les unes avec les autres et prônait une coopération entre les milieux d'affaires et l'État pour la conquête des marchés; bien qu'elle raisonnât à l'échelle globale, elle ne délaissait pas le continent européen, l'un de ses objectifs étant l'établissement d'une aire économique sous domination allemande en Europe centrale. Dans ce projet, les provinces russes et austro-hongroises étaient conçues comme un réservoir de matières premières pour les industries allemandes, qui leur enverraient, en retour, leurs produits manufacturés ${ }^{(2)}$. De ce point de vue-là, David Blackbourn le rappelle, «le véritable équivalent allemand de l'Inde et de l'Algérie n'était pas le Cameroun: c'était la Mitteleuropa ${ }^{(3)}$.

* Attaché Temporaire d'Enseignement et de Recherche en histoire contemporaine à l'Université de Strasbourg.

1 Woodruff D. Sмiтн, «The Ideology of German Colonialism, 1840-1906», Journal of Modern History, 46 (1974), p. 641-662.

2 Ibid., p. 644, 657.

3 David Blackbourn, "Das Kaiserreich transnational: Eine Skizze», in: S. Conrad et J. Osterhammel (dir.), Das Kaiserreich transnational, Göttingen, Vandenhoeck \& Ruprecht, 2004, p. 322; sur l'histoire des projets allemands dans la Mitteleuropa, cf. Jörg Brechtefeld, Mitteleuropa and German Politics: 1848 to the Present, New York, St. Martin's Press, 1996; Friedrich Lenz, Deutsche Mitteleuropa-Pläne, 1815-1945: eine Studie zum modernen Imperialismus und zur Formation der bürgerlichen Gesellschaft, Wiesbaden-Amöneburg, Verlag Edmund Steinschulte, 2008. 
L'analyse vaut en particulier si l'on s'intéresse à l'exploitation d'une ressource naturelle comme le bois. Contrairement à celui du coton ${ }^{(4)}$, l'empire allemand du bois se situait presque intégralement en Europe. En 1913, la Russie et l'Autriche-Hongrie fournissaient $77 \%$ des importations allemandes de bois d'œuvre et de pâte à papier, le reste provenant pour l'essentiel de Scandinavie (12\%) et des États-Unis (7 \%); quant à l'Afrique de l'Ouest, où se trouvait la colonie du Cameroun, elle contribuait à moins de $1 \%$ du total ${ }^{(5)}$. En partant de ce constat, l'article se propose d'examiner l'économie forestière de la Galicie autrichienne, région devenue au début des années 1890 l'une des plus importantes zones d'approvisionnement en bois de l'Empire allemand. Dans cette région de $78500 \mathrm{~km}^{2}$ intégrée à l'Empire des Habsbourg lors des partages de la Pologne de la fin du XVIII ${ }^{e}$ siècle, les forêts, longtemps restées peu exploitées en raison du manque de voies de communication, firent au tournant du $\mathrm{XX}^{\mathrm{e}}$ siècle l'objet d'une pénétration commerciale allemande qui déboucha sur une exploitation à grande échelle de leurs ressources ligneuses. En quoi cet épisode de l'histoire des forêts galiciennes partage-t-il par sa forme, mais aussi par ses conséquences économiques, environnementales et sociales, certains traits caractéristiques des pratiques prédatrices des puissances (néo)coloniales européennes en Amérique, en Afrique ou en Asie?

\section{Le capital allemand à l'assaut des forêts galiciennes}

Dans Globalization and the Race for Resources (2005), Stephen G. Bunker et Paul S. Ciccantell ont développé la thèse selon laquelle le pouvoir global repose sur le contrôle des ressources naturelles dont dépend la croissance ${ }^{(6)}$. La montée en puissance de l'Allemagne sur la scène mondiale durant les années 1890-1914 s'inscrit dans cette dynamique, l'un des objectifs de la Weltpolitik ayant été la lutte pour l'accès aux matières premières dans le contexte d'une mondialisation des échanges ${ }^{(7)}$. Dans l'histoire du commerce du bois, le $\mathrm{XIX}^{\mathrm{e}}$ siècle a représenté une période de rivalités entre les États, à travers une série de conflits douaniers, mais aussi entre capitalistes, qui ont dû surmonter de nombreux obstacles pour maintenir leurs profits ${ }^{(8)}$. Contrairement à une idée reçue, l'industrialisation de l'Europe n'avait pas entraîné une baisse de la consommation de bois, et ce en dépit de l'utilisation croissante du charbon ${ }^{(9)}$. Vers

4 Thaddeus Sunseri, «The Baumwollfrage: Cotton Colonialism in German East Africa», Central European History, 34 (2001), p. 31-51; Andrew Zimmerman, "A German Alabama in Africa: The Tuskegee Expedition to German Togo and the Transnational Origins of West African Cotton Growers », American Historical Review, 110 (2005), p. 1362-1398.

5 Walter Hedler, Deutschlands Forst- und Nutzholzwirtschaft in und nach dem Weltkriege, Stuttgart, 1921, p. 24-25.

6 Stephen G. Bunker et Paul S. Ciccantell, Globalization and the Race for Resources, Baltimore, Johns Hopkins University Press, 2005.

7 Sur l'histoire du Kaiserreich dans la mondialisation, cf. Sebastian ConRad, Globalisation and the Nation in Imperial Germany, Cambridge/New York, Cambridge University Press, 2010; Cornelius Torp, The Challenges of Globalization: Economy and Politics in Germany, 1860-1914, New York, Berghahn Books, 2014.

8 Sing C. CHew, Logs for Capital: The Timber Industry and Capitalist Enterprise in the Nineteenth Century, Westport, Greenwood Press, 1992, p. 20.

9 Iñaki Iriarte-GoÑ et María-Isabel AyudA, «Not Only Subterranean Forests: Wood Consumption and Economic Development in Britain (1850-1938)», Ecological Economics, 77 (2012), p. 176-184. 
1900, l'Allemagne consommait 60 millions de stères de bois de feu, un tiers de plus qu'en $1872^{(10)}$. Avec l'urbanisation, les besoins en bois de charpente avaient explosé(11). Il en allait de même pour les bois ronds (poteaux, étais de mine) et la pâte à papier.

Or comme cette hausse de la consommation survenait à un rythme plus rapide que la croissance des rendements sylvicoles, on craignait de plus en plus une pénurie internationale de bois ${ }^{(12)}$. Pour assurer son développement, l'Allemagne recourait depuis plusieurs décennies à des importations massives ${ }^{(13)}$. En 1864, le pays était devenu importateur net; le volume des livraisons depuis l'étranger s'accrut encore considérablement au tournant du $\mathrm{XX}^{\mathrm{e}}$ siècle, passant de 3,3 millions de tonnes en 1880 à 6,9 en 1906 , date à laquelle elles atteignirent leur plus haut niveau ${ }^{(14)}$. Elles couvraient alors $41 \%$ de la consommation intérieure et représentaient, avec une facture de 250 millions de marks, le troisième poste dans les importations de l'Empire allemand, derrière les produits agricoles et textiles ${ }^{(15)}$.

Conséquence de la politique de libéralisation des échanges adoptée sous le chancelier Caprivi (1890-1894), cette évolution manifestait l'hégémonie commerciale de l'Empire allemand en Europe centrale ${ }^{(16)}$. En 1891 puis en 1895, des traités de commerce austro-allemands réduisirent les droits sur le bois, ce qui provoqua une envolée des exportations de l'Autriche-Hongrie ${ }^{(17)}$. La Galicie fut la province la plus touchée par cet essor. Couvrant une surface d'environ 2 millions d'hectares, ses forêts regorgeaient encore d'immenses réserves au début des années 1890, en particulier sur les contreforts de Carpates, dans le massif des Beskides et la vallée du Dniestr ${ }^{(18)}$. En 1885, on avait achevé la construction d'une voie de chemin de fer, la Transversalbahn, qui ouvrait aux investisseurs environ 200000 ha de forêts ${ }^{(19)}$. Desservant la lisière des Carpates, cette ligne offrait une alternative au flottage des bois vers Danzig, liée à

10 «Wie deckt Deutschland seinen Nutzholzbedarf?», Deutsche Forst-Zeitung, 19 (1904), p. 4-7.

11 Otto Pflanze, Bismarck and the Development of Germany, II: The Period of Consolidation, 1871-1880, Princeton, Princeton University Press, 1990, p. 284.

12 Christian Lotz, «Expanding the Space for Future Resource Management: Explorations of the Timber Frontier in Northern Europe and the Rescaling of Sustainability during the Nineteenth Century", Environment and History, 21 (2015), p. 257-279, ici p. 265.

13 Bernd Stefan Grewe, «Das Ende der Nachhaltigkeit? Wald und Industrialisierung im 19. Jahrhundert», Archiv für Sozialgeschichte, 43 (2003), p. 61-79.

14 Max Endres, Handbuch der Forstpolitik, mit besonderer Berücksichtigung der Gesetzgebung und Statistik, Berlin, Springer, 1922 (2 éd.), p. 586-587.

15 Max Endres, Die Leistungsfähigkeit der Forstwirtschaft, Munich, Wolf, 1907, p. 18; Verhandlungen des königlichen Landes-Ökonomie-Kollegiums vom 5. bis 8. Februar 1908. I. Tagung der XI. SitzungsPeriode, Berlin, Parey, 1908, p. 524-525.

16 Irena Pietrzak-Pawıowska, «Pozycja ziem polskich w międzynarodowej wymianie gospodarczej na przełomie XIX i XX wieku (La position des terres polonaises dans les échanges économiques internationaux au tournant des $\mathrm{XIX}^{\mathrm{e}}$ et $\mathrm{XX}^{\mathrm{e}}$ siècles) », in: VIII Congrès des historiens polonais à Cracovie, $14-17$ septembre 1958. VI. Histoire économique et sociale, Société polonaise d'histoire, 1960, p. 367-396, ici p. 383.

17 Franz von Mammen, Deutschlands und Oesterreich-Ungarns Holzzollpolitik vor, während und nach dem Weltkriege, Dresden, Globus, 1916.

18 Seweryn Tyc, «Luźne uwagi z dziedziny handlu i przemysłu drzewnego (Remarques en vrac sur le secteur du commerce et de l'industrie du bois)», Sylwan, 10 (1892), p. 62-75.

19 Adam Schwappach, «Eine Studienreise nach Schlesien, Galizien und Ungarn», Allgemeine Forstund Jagd-Zeitung, 61 (1885), p. 75-76. 
l'orientation nord-sud des principaux cours d'eau (Vistule, San, Bug), et permettait leur acheminement vers l'Empire allemand via la Silésie prussienne ${ }^{(20)}$.

Comme le note Taddeus Sunseri, la «forêt primaire» (Urwald) était alors conçue par les forestiers et les industriels comme un espace sauvage et improductif à mettre en valeur ${ }^{(21)}$. En Afrique comme en Europe, cette représentation offrait aux firmes d'exploitation du bois un argumentaire pour légitimer leurs stratégies d'expansion. L'heure était au désenclavement des forêts et à leur pénétration commerciale, deux idées que résume le terme allemand Erschließung, maître-mot de la conquête coloniale ${ }^{(22)}$. C'est précisément dans cet esprit que s'exprima le professeur de sylviculture Max Endres à l'occasion de sa nomination au poste de recteur à l'Université Louis-etMaximilien de Munich, en 1907:

«Ce n'est pas la force humaine qui apprivoise [la forêt primaire], mais le capital de l'entrepreneur. Celui qui veut intégrer les coupes de bois des complexes forestiers reculés au marché mondial doit bâtir des logements pour ses employés, fonder des colonies de travailleurs, ériger des scieries, aménager des chemins de roulement et des chemins de fer, rendre les ruisseaux forestiers et les fleuves navigables, veiller à l'évacuation ou à la destruction de la matière ligneuse non exploitable et nouer des relations commerciales à tous les points cardinaux. [...] Seules les sociétés de commerce ou les industriels disposant du soutien du grand capital peuvent ici ouvrir la voie. Leur performance constitue pour la sylviculture un bénéfice semblable à l'œuvre colonisatrice des seigneuries [...] médiévales " ${ }^{(23)}$.

En parlant d'«œuvre colonisatrice», Endres pensait peut-être à l'expansion allemande dans l'est de l'Europe (Ostsiedlung), période historique devenue un véritable mythe national au XIX ${ }^{\mathrm{e}}$ siècle ${ }^{(24)}$ et dont l'un des «hauts faits» avait été d'importants défrichements ${ }^{(25)}$. À la manœuvre dans la destruction des forêts sauvages se trouvaient cette fois non pas les seigneurs féodaux, mais les grands magnats du bois de l'ère du capitalisme financier.

20 Sur le caractère monodirectionnel du commerce des bois dans les régions montagneuses avant le développement du chemin de fer, cf. C. Lotz, «Expanding the Space» (note 12), p. 262.

21 Thaddeus Sunseri, «Exploiting the Urwald: German Post-Colonial Forestry in Poland and Central Africa, 1900-1960», Past and Present, 214 (2012), p. 305-342, ici p. 305.

22 Sur le cas de l'Afrique, cf. Dirk van LAAK, Imperiale Infrastruktur: deutsche Planungen für eine Erschließung Afrikas 1880 bis 1960, Paderborn, Schöningh, 2004.

23 "Nicht die menschliche Kraft bezwingt [den Urwald], sondern nur das Kapital des Unternehmers. Wer die Holzschläge entlegener Waldkomplexe dem Weltmarkt erschließen will, muß Beamtenwohnungen schaffen und Arbeiterkolonien gründen, Sägewerke errichten, Rollbahnen und Eisenbahnen bauen, Waldbäche und Flüße floßbar machen, für die Beseitigung oder Vernichtung des nicht verwertbaren Holzmaterials sorgen und Handelsbeziehungen nach allen Windrichtungen hin anknüpfen. [...] Nur Handelsgesellschaften oder Industrielle mit Rückendeckung des Großkapitals können hier freie Bahn schaffen; ihre Wirksamkeit ist ein Verdienst um die Waldkultur, vergleichbar mit der Kolonisationstätigkeit der [...] Grundherrschaften des Mittelalters. » M. Endres, Die Leistungsfähigkeit (note 15), p. 25-26.

24 Wolfgang Wippermann, Der «deutsche Drang nach Osten»: Ideologie und Wirklichkeit eines politischen Schlagwortes, Darmstadt, Wissenschaftliche Buchgesellschaft, 1981; Tzu-hsin Tu, Die Deutsche Ostsiedlung als Ideologie bis zum Ende des Ersten Weltkriegs, Kassel, Kassel University Press, 2009.

25 L'historien Karl Lamprecht, par exemple, présentait l'espace germanique comme le centre d'impulsion des défrichements en Europe centre-orientale à partir du XII ${ }^{\mathrm{e}}$ siècle. Cf. Karl Lamprecht, Deutsche Geschichte: Urzeit und Mittelalter, Fribourg-en-Brisgau, Heyfelder, 1906 (3éd.), p. 370-371. 
En Galicie, le commerce extérieur du bois était contrôlé par des marchands étrangers, prussiens pour l'essentiel, dans une moindre mesure hongrois et autrichiens, alors que la région était surtout peuplée de Polonais et d'Ukrainiens. Vers 1900, une poignée de compagnies basées à Berlin et en Haute Silésie dominaient le secteur : le Berliner HolzComptoir, David Francke \& Fils, la Oberschlesische Holzindustrie, Schlesinger \& Fils et quelques autres. Elles avaient rapidement remplacé les entreprises plus petites gérées par des juifs galiciens qui dominaient le marché du bois jusqu’alors ${ }^{(26)}$. Ces firmes, en partie sociétés par actions, acquéraient soit les forêts (bois sur pied et sol compris), soit un droit d'exploitation pour plusieurs années. En 1897 par exemple, David Francke \& Fils et le Berliner Holz-Comptoir achetèrent pour la somme astronomique de 5 millions de marks le domaine de Mokrzyszów (8 595 ha de forêts), situé près de Tarnobrzeg ${ }^{(27)}$. Les opérations de ce type se multiplièrent durant les années suivantes. En 1911, la direction de Schlesinger \& Fils fit l'acquisition de forêts publiques dans les Beskides orientales, où elle fit construire des scieries et une voie ferrée de 60 kilomètres pour les relier aux zones de coupe ${ }^{(28)}$.

Faisant ainsi tomber les derniers des remparts d'inaccessibilité, les capitaux et le chemin de fer permirent aux compagnies allemandes de monter à «l'assaut» des forêts galiciennes. Cette expression, empruntée à l'historien canadien Arthur Lower ${ }^{(29)}$, permet de souligner le caractère brutal et rapide de la pénétration commerciale allemande dans l'économie forestière de Galicie au tournant du $\mathrm{XX}^{\mathrm{e}}$ siècle. Dans cette étude de géographie historique, Lower montrait comment les forêts canadiennes, longtemps exploitées au profit de la Grande-Bretagne ${ }^{(30)}$, avaient été au milieu du XIX ${ }^{\mathrm{e}}$ siècle entraînées dans l'orbite du marché américain, ce qui avait conduit au pillage de leurs ressources. Cette mise sous tutelle était la conséquence à la fois du traité de Réciprocité (1854), qui ouvrait le marché canadien au commerce américain, et d’une réorientation des flux induite par le passage d'un transport des bois par flottage sur le réseau hydrographique à un transport par le chemin de fer. Les forêts galiciennes connurent un destin semblable - l'Empire allemand jouant ici le rôle des États-Unis - à la suite d'une extension du réseau ferré et de la signature des accords de 1891 et 1895, facteurs auxquels il faudrait ajouter l'épuisement des ressources en bois dans la Pologne russe, qui poussa également les industriels allemands à se tourner vers les réserves de Galicie ${ }^{(31)}$.

26 Ignacy SchIPeR, Dzieje handlu żydowskiego na ziemiach polskich (Histoire du commerce juif sur les terres polonaises), Varsovie, Związek Kupców, 1937, p. 446, 450.

27 Jahrbuch der Berliner Börse. Ein Nachschlagebuch für Bankiers und Kapitalisten, Berlin, Berliner Actionair, 1899, p. 533.

28 «Forstverkäufe in Österreich-Ungarn», Deutsche Forst-Zeitung, 26 (1911), p. 130.

29 Arthur R.M. Lower, The North American Assault on the Canadian Forest: A History of the Lumber Trade Between Canada and the United States, Toronto, Ryerson Press, 1938.

30 Sur ce sujet, cf. Arthur R. M. Lower, Great Britain's Woodyard. British America and the Timber Trade, 1763-1867, Montreal, McGill-Queen's University Press, 1973; Graeme Wynn, Timber Colony: A Historical Geography of Early Nineteenth Century New Brunswick, Toronto, University of Toronto Press, 1981.

31 Käthe Schirmacher, Les Travailleurs du bois de Danzig, Paris, A. Rousseau, 1905, p. 45-46. Ce glissement des activités d'extraction de bois vers le sud s'inscrit dans le déplacement de la «frontière du bois d'œuvre» (timber frontier) en Europe centrale. Sur ce phénomène en Europe du Nord, cf. Jörgen BJÖRKLUnd, «Exploiting the Last Phase of the North European Timber Frontier for the International Market 1890-1914: An Economic-Historical Approach», in: Mauro Agnoletti et Steven 


\section{Le commerce de bois entre la Galicie et l'Empire allemand}

En 1885, la province ne contribuait encore que faiblement aux exportations de bois de l'Autriche-Hongrie vers l'Allemagne, avec 44000 tonnes, soit à peine 8,8\% du total. Le chiffre connut ensuite une rapide augmentation, pour atteindre près de $30 \%$ en 1898. Cette année-là, 580000 tonnes de bois de construction, de bois de chauffage et de pâte à papier partirent vers l'Allemagne par le chemin de fer (cf. fig. 1).

Fig. 1 : Les exportations de bois de la Galicie vers l'Allemagne d'après les statistiques ferroviaires $(1885-1898)^{(32)}$

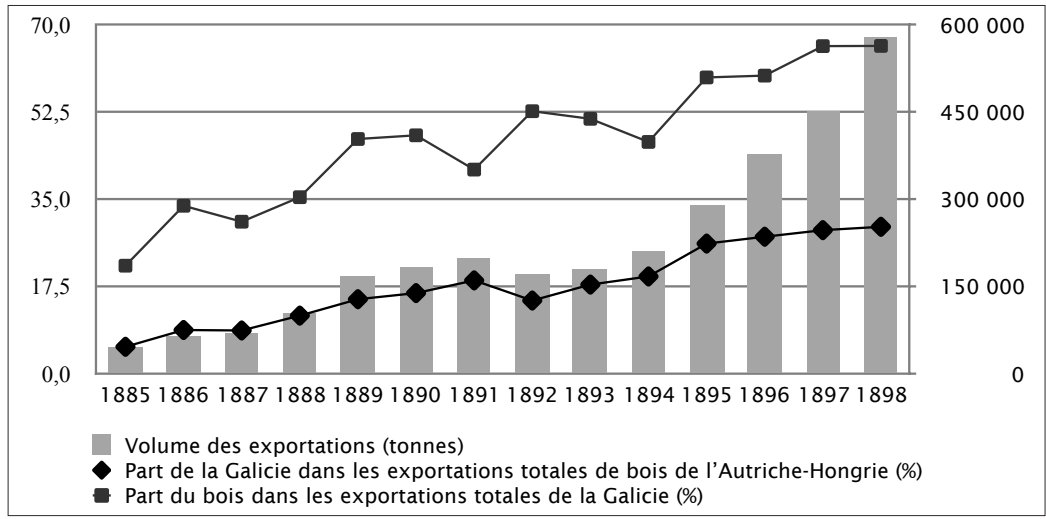

Ces données montrent également la part écrasante ${ }^{(33)}$ que les produits ligneux prirent dans le commerce extérieur de la Galicie durant cette période. En 1897, ils représentaient en poids plus des deux tiers $(65,8 \%)$ des exportations vers l'Empire allemand, contre à peine un cinquième $(21,7 \%)$ quinze ans plus tôt. Il faut ajouter à cela que les flux vers d'autres pays étaient minimes: en 1913, 78 \% des bois de construction exportés partaient vers l’Allemagne, loin devant la Bohême (11,6 \%), la Hongrie $(5,4 \%)$ et l'Autriche $(5,1 \%)^{(34)}$.

Cette tendance à l'extraversion était aussi une spécialisation qualitative, car la Galicie exportait principalement des bois non transformés. Vers 1900, les tarifs douaniers allaient de 20 marks/wagon pour les bois bruts à 80 marks pour les bois sciés. Mais

ANDERson (dir.), Forest History: International Studies on Socio-economic and Forest Ecosystem Change, Wallingford, CABI, 2000, p. 171-184; C. Lotz, «Expanding the Space» (note 12).

32 Calculé d’après Kornel PAYgert, «Podstawy do określenia żądań Galicyi na polu polityki handlowej (Fondements servant à déterminer les besoins de la Galicie dans le domaine de la politique commerciale)», Przegląd Polski, 146 (1902), p. 467-497, ici p. 475; M. Endres, Handbuch (note 14), p. 591.

La part du bois dans les exportations totales était en réalité plus élevée car ces chiffres ne prennent en compte que les produits expédiés par le chemin de fer, alors que des quantités importantes de bois partaient également vers la Prusse par flottage. En 1903, 60895 tonnes arrivèrent ainsi sur la Vistule au poste frontalier de Schillno, ce qui représentait $12 \%$ du total importé par cette voie. Cf. Władysław Piechowski, «Wisła jak droga komunikacyjna i handlowa (La Vistule comme voie de communication et de commerce)», Ekonomista, 6 (1906), p. 81-100, ici p. 86-89.

34 Leon Władysław Biegeleisen, Stan ekonomiczny Małopolski na podstawie bilansu handlowego (L'état économique de la Petite-Pologne sur la base de son bilan commercial), Varsovie, 1921, p. 346-361. 
comme le faisait remarquer l'économiste galicien Kornel Paygert, le tarif appliqué aux bois sciés était surévalué: si l'on s'en était tenu à prendre en compte le supplément de valeur ajoutée, il n'aurait dû être que de 36 marks $^{(35)}$. Les firmes allemandes propriétaires de scieries en Galicie pouvaient supporter ces tarifs grâce à leurs capacités d'investissement, qui leur permettaient de réaliser des économies d'échelles ${ }^{(36)}$. Ce n'était pas le cas des petits artisans et industriels galiciens - scieurs, menuisiers, parqueteurs, ébénistes - qui représentaient la majorité des 16500 établissements actifs dans ce secteur ${ }^{(37)}$. Constatant que le bois galicien se vendait moins cher à Berlin qu'à Cracovie ou à Lwów, l'économiste Roman Woyczyński analysa en 1911 la hausse de la demande locale suite à la raréfaction du matériau, massivement parti à l'exportation ${ }^{(38)}$. De cette situation résultait un sous-équipement en industries de première transformation, pourtant rentables et faciles à fabriquer ${ }^{(39)}$. En Prusse au contraire les usines prospéraient grâce à l'afflux de bois galicien. Woyczyński prenait l'exemple de Ziegenhals, petite ville frontalière de Silésie prussienne; l'usine Tillgner \& Co, ouverte en 1883, y fabriquait tous types de produits, de la cellulose aux barils, ensuite revendus en Galicie. Inondée de produits allemands bon marché, la région avait vu ses industries locales péricliter. Faute de scieries, même les grands domaines devaient se contenter de vendre des bois bruts, de telle sorte que leurs bénéfices stagnaient ${ }^{(40)}$. On est frappé de voir qu'en 1913, la Galicie importait d'énormes quantités de produits de fabrication pourtant simple, comme des charrettes, des tonneaux, des balais et des brosses (cf. fig. 2).

Bien qu'elle disposât de plusieurs atouts pour avoir une industrie du bois performante, en premier lieu une abondance de matière première et de main-d'œuvre, la Galicie se retrouvait contrainte, du fait de sa dépendance technologique et financière vis-à-vis des compagnies allemandes, d'exporter des bois bruts à bas prix (environ 82 couronnes/tonne) et de payer cher des produits manufacturés importés (711 couronnes/tonne).

Cette structure des échanges révèle la position périphérique de la Galicie au sein du «système-monde» tel que le décrit Immanuel Wallerstein ${ }^{(41)}$. Selon cette approche, le processus d'accumulation du capital en marche depuis le $\mathrm{XVI}^{\mathrm{e}}$ siècle repose avant tout sur une division du travail entre différentes régions du monde ${ }^{(42)}$. Dans le «noyau»

35 K. Paygert «Podstawy» (note 32), p. 289.

36 Sur l'importance des économies d'échelle dans l'expansion du système capitaliste, cf. BunKer/ CICCANTELL, Globalization (note 6).

37 74,5 \% étaient des entreprises individuelles et 22,7 \% employaient moins de 5 personnes. Cf. J. BrodA, Historia leśnictwa w Polsce (Histoire de la sylviculture en Pologne), Poznań, Akademia Rolnicza im. Augusta Cieszkowskiego, 2000, p. 67.

38 Roman Woyczyński, «Przemysł drzewny w Galicyi a gospodarstwa leśne (L’industrie du bois en Galicie et les exploitations forestières)», Sylwan, 29 (1911), p. 388-391.

39 On observe le même paradoxe aux $\mathrm{XVI}^{\mathrm{e}}-\mathrm{XVII}^{\mathrm{e}}$ siècles: devenue la réserve stratégique de bois de l'Europe, la Pologne avait fini par manquer de cette matière première pour son propre développement. Cf. Andrzej Wy robisz, «La crise de combustibles dans l'industrie polonaise au tournant du XVI ${ }^{\mathrm{e}}$ et XVII ${ }^{\mathrm{e}}$ siècle», Studia historiae oeconomicae, 8 (1973), p. 259-269.

40 R. Woyczyński, «Przemysł drzewny» (note 38).

41 Immanuel Wallerstein, Capitalist Agriculture and the Origins of the European World-Economy in the Sixteenth Century, New York, Academic Press, 1974.

42 Ibid., p. 349. 
(core) du système, on observe un développement des activités les plus productives, tandis que la périphérie se spécialise dans la monoculture et l'extraction de matières premières $^{(43)}$. Le secteur du bois en livre ici un exemple emblématique. Il confirme le constat général établi par Klemens Kaps, selon lequel l'économie galicienne a été entre 1772 et 1914 sujette à une "périphérisation" au sein du système-monde, ce qui s'est traduit par une désintégration de son tissu industriel et la dégradation de ses termes de l'échange ${ }^{(44)}$. La balance commerciale pour les produits ligneux était certes excédentaire (cf. fig. 2), mais depuis 1900, les prix à l'export des grumes équarries ou des bois sciés stagnaient autour de 60-80 marks/tonne (cf. fig. 3), à cause de l'absence totale de planification des coupes au niveau provincial et d'une tendance à la surproduction de bois pour le marché extérieur ${ }^{(45)}$.

Fig. 2: Bois et produits manufacturés en bois dans le commerce extérieur de la Galicie en $1913^{(46)}$

\begin{tabular}{|r|r|r|r|r|}
\hline \multirow{2}{*}{ Produits } & \multicolumn{2}{|c|}{ Exportations } & \multicolumn{2}{c|}{ Importations } \\
\cline { 2 - 5 } & Tonnes & Couronnes & \multicolumn{1}{c|}{ Tonnes } & Couronnes \\
\hline Bois de construction & 369577 & 30330812 & 1899 & 162072 \\
\hline Bois de chauffage & 21776 & 398322 & 3766 & 67642 \\
\hline Arbres fruitiers & - & - & 19 & 11592 \\
\hline Poteaux & 4680 & 150639 & - & - \\
\hline Plants & 44 & 32800 & 82 & 48818 \\
\hline Arbres de Noël & 296 & 222000 & - & - \\
\hline Planches & - & - & 168 & 29480 \\
\hline Meubles & 184 & 330841 & 1869 & 4132655 \\
\hline Ustensiles domestiques & - & - & 81 & 35403 \\
\hline Coffrages & - & - & 126 & 39915 \\
\hline Charrettes & 95 & 770390 & 338 & 2719046 \\
\hline Balais et brosses & - & - & 112 & 114511 \\
\hline Tonneaux & 2607 & 613920 & 6572 & 1659678 \\
\hline Total & 399364 & $\mathbf{3 2 9 5 6 3 8 0}$ & 18280 & 13004655 \\
\hline & 105 & 136656 & 3248 & 3983843 \\
\hline
\end{tabular}

43 Ibid., p. 101.

44 Klemens KAPs, Ungleiche Entwicklung in Zentraleuropa: Galizien zwischen überregionaler Verflechtung und imperialer Politik (1772-1914), Vienne, Böhlau, 2015.

45 Le même constat vaut pour l'économie du pétrole. Cf. Alison F. Frank, Oil Empire: Visions of Prosperity in Austrian Galicia, Cambridge, Harvard University Press, 2005, p. 140-172.

46 L. Pączewski, Lasy, przemyst $i$ handel drzewny w Polsce (Les forêts, l'industrie et le commerce du bois en Pologne), Varsovie, Instytut Wydawniczy Bibljoteka Polska, 1924, p. 163-164. 
Fig. 3: Prix des bois exportés par la Galicie vers l'Allemagne d'après les statistiques ferroviaires (1900-1913) ${ }^{(47)}$

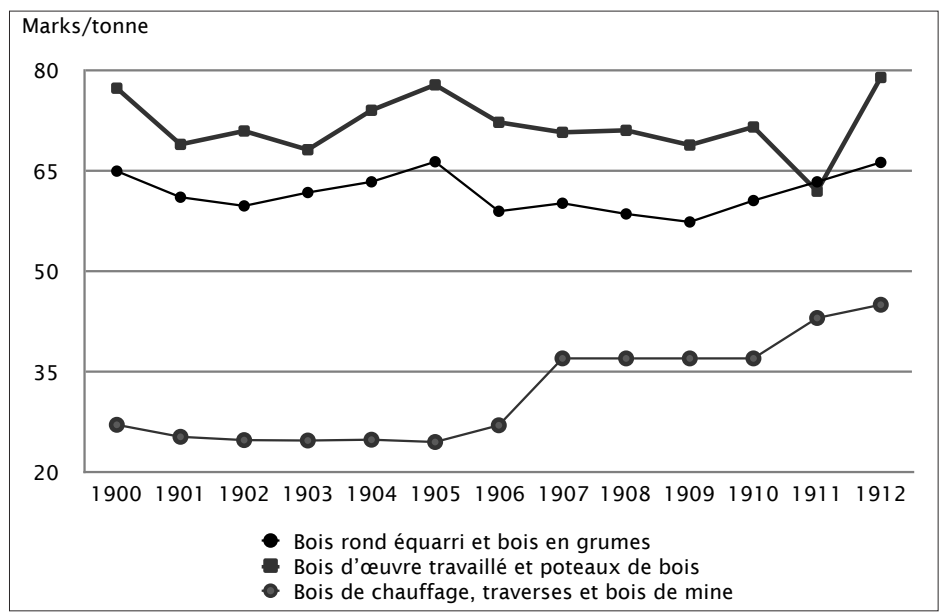

Le bénéfice des exportations de bois pour la société galicienne était donc largement amputé par l'augmentation tendancielle du coût des importations d'autres produits ${ }^{(48)}$, mais aussi et surtout par les externalités négatives qu'impliquaient les prélèvements à grande échelle dans les forêts.

\section{Le coût environnemental et social de l'extraction des bois}

Comme l'a montré Stephen G. Bunker dans son ouvrage intitulé Underdeveloping the Amazon (1985), et à sa suite toute une série de chercheurs comme Alf Hornborg, John R. McNeill et Juan Martinez Alier, les flux mondiaux de matière (et donc d'énergie) affectent de façon différenciée les milieux naturels des centres et des périphéries, au détriment de ces dernières, qui se voient forcées de supporter les fardeaux sociaux et environnementaux exportés par les centres ${ }^{(49)}$. En d'autres termes, les matières premières exportées par les pays périphériques le sont à des prix qui ne couvrent pas l'intégralité des dégâts induits par leur production ${ }^{(50)}$. En Galicie, comme dans d'autres périphéries du système-monde, les formes de prélèvement du bois d'œuvre s'inscrivaient dans ce que Bunker appelle une «économie extractive», fondée sur la minimisation des coûts d'exploitation et une logique court-termiste ${ }^{(51)}$. La préservation des écosystèmes n'était

47 Calculé d'après L. W. Biegeleisen, Stan ekonomiczny Małopolski (note 34), p. 390-391, 417.

48 Sur la dégradation des termes de l'échange de la Galicie sur le long terme, cf. K. Kaps, Ungleiche Entwicklung in Zentraleuropa (note 44).

49 Stephen G. Bunker, Underdeveloping the Amazon. Extraction, Unequal Exchange and the Failure of the Modern State, Chicago, The University of Chicago Press, 1985; Alf Hornborg, John R. McNeILL et Juan Martínez Alier, Rethinking Environmental History: World-System History and Global Environmental Change, Lanham, AltaMira Press, 2007.

50 Juan Martinez-Alier et Maite Cabeza-Gutés, «L'échange écologiquement inégal», in: Michel Damian et Jean-Christophe Graz (dir.), Commerce international et développement soutenable, Paris, Economica, 2001, p. 159-185, ici p. 175.

51 S. G. Bunker, Underdeveloping the Amazon (note 49). 
pas la priorité des firmes allemandes et les propriétaires avec lesquels elles traitaient ne s'empressaient pas de reboiser leurs terrains après les coupes, alors que la loi forestière du 3 décembre 1852 le leur imposait, sous peine d'amendes ${ }^{(52)}$.

Les relations entre la firme Goldstein \& Frères de Breslau et le comte Tarnowski illustrent ce phénomène. En 1899, un contrat de 55050 thalers fut signé pour des coupes sur le domaine de Tarnobrzeg; les acheteurs avaient deux ans pour abattre les arbres et débarrasser les terrains des bois rémanents afin qu'ils puissent être remis en culture $^{(53)}$. Mais comme ils tardaient à le faire, le comte entra en conflit avec eux ${ }^{(54)}$; en parallèle, l'inspecteur des forêts de Tarnów le pressait de reboiser au plus vite l'un de ses terrains, demande qui traînait déjà depuis quatre ans ${ }^{(55)}$. Sur le domaine de Lańcut, le comte Potocki n'avait lui non plus pas entrepris le reboisement des parcelles exploitées par les marchands ${ }^{(56)}$. En 1903-1904, la starostie et le gouverneur le rappelèrent à l'ordre à plusieurs reprises ${ }^{(57)}$.

Durant les années 1900, les cas de coupes illégales et d'absence de reboisement furent souvent rendus publics dans la presse, car ils compromettaient la régénération des stocks sur pied. L'Ami du peuple, organe du PSL (Polskie Stronnictwo Ludowe), parti agrarien de Galicie, publia en 1903 la liste de forêts dévastées par les marchands dans les arrondissements de Tarnobrzeg, Ropczyce et Mielec ${ }^{(58)}$. Il citait les noms des propriétaires concernés et l'impact du déboisement sur l'environnement : érosion des sols, formation de dunes de sable qui menaçaient les cultures. Beaucoup d'arbres abattus étaient immatures, c'est-à-dire âgés de moins de 40 ans, parfois même de moins de 15 ans car à cette époque on vendait de jeunes épicéas utilisés en Prusse comme sapins de Noël ${ }^{(59)}$. Lors d'une intervention à la Diète de Lwów en 1904, le député agrarien Andrzej Szponder s'en prit violemment aux responsables de ce pillage des forêts:

«Des bruissantes et séculaires forêts polonaises, il ne reste plus de trace et de souvenir que dans les romans et la poésie, car les Prussiens ont tout arraché et ont tout emporté. Bien que les principaux coupables de la déforestation dans notre pays soient les propriétaires des forêts ainsi que divers spéculateurs malhonnêtes, il est difficile de ne pas reconnaître le fait douloureux que dans tous les malheurs qui s'abattent sur nous, mis à part les catastrophes naturelles, le Prussien occupe toujours une place de choix» ${ }^{(60)}$.

Jules Madelin, Les restrictions légales au droit de propriété forestière privée en France, en Allemagne, en Autriche-Hongrie et en Suisse, Paris, A. Rousseau, 1905, p. 161-162.

53 Archiwum Narodowe w Krakowie (ANwK) 639 n² 2331, p. 217.

Ibid., p. 231-232.

ANwK 639 n²298, p. 7-8.

56 Archiwum Główny Akt Dawnych (AGAD) 350 nº 564.

57 Ibid., p. 79-83.

58 Przyjaciel Ludu, 02.08.1903 et 23.03.1903.

59 Sur ce sujet, cf. Jawad DAHEur, «Exporting Environmental Burdens into the Central-European Periphery: Christmas Tree Trade and Unequal Ecological Exchange between Germany and Habsburg Galicia around $1900 »$, Historyka. Studia Metodologiczne (à paraître).

60 «O szumiących, stuletnich borach polskich pozostanie ślad i wspomnienie tylko w powieściach i poezyi, bo Prusacy wszystko wycięli i zabrali. Jakkolwiek główna wina za ogołocenie z lasów kraju naszego spada na właścicieli tychże i różnych nieuczciwych spekulantów, to jednak trudno się powstrzymać od zaznaczenia bolesnego faktu, że we wszystkich nieszczęściach, jakie na nas spadają, krom klęsk elementarnych, Prusak zajmuje zawsze wybitne miejsce.» Sprawozdanie Stenograficzne $z$ Rozpraw Galicyjskiego Sejmu Krajowego, Lwów, 1904, Kadencja VIII, sesja II, pos. 5, p. 188. 
Au-delà de ses excès langagiers, ce discours reposait sur une réalité que confirment les chiffres du commerce avec l'Allemagne. La ponction était considérable. Au début des années 1900, les exportations par le chemin de fer, environ 1,4 million de stères, représentaient $18 \%$ de l'accroissement naturel et $30 \%$ de la récolte ${ }^{(61)}$. Des chiffres aussi élevés ne laissaient pas les contemporains indifférents. Ladislaus Krzanowski, administrateur des chemins de fer, portait rétrospectivement un jugement sévère sur l'ouverture de la Transversalbahn:

«Un regard jeté à travers la fenêtre du coupé suffit à prouver que là où durant les premières années après la mise en service on trouvait encore de vieilles forêts à proximité du chemin de fer, le voyageur ne voit plus maintenant que des monts glabres, à la végétation clairsemée [...]. Une question vient inconsciemment au cheminot: et maintenant? Lorsque nous aurons exporté le bois et le pétrole - et en ce qui concerne le bois il semble que l'on en arrive bientôt au bout -, quoi d'autre allons-nous encore "exporter" ?...»"

Krzanowski le rappelle, l'une des caractéristiques de l'économie extractive est une certaine prédominance du court terme. Or comme Bunker le montre pour l'Amazonie, cette façon de concevoir l'exploitation des ressources naturelles est en rupture totale avec l'économie de subsistance antérieure ${ }^{(63)}$. En Galicie, la transition énergétique du bois au charbon était à peine entamée au début du $\mathrm{XX}^{\mathrm{e}}$ siècle; l'utilisation du charbon de bois était très répandue, y compris dans l'industrie lorsque des forêts étaient proches ${ }^{(64)}$. L'envolée des prix du bois à partir de 1895, dont le cas de Cracovie donne une illustration (cf. fig. 4), fut donc violemment ressentie par une société dont le mode de vie dépendait largement de ce matériau.

Le profil des courbes révèle l'impact des accords douaniers à travers une envolée des prix du bois de chauffage dur et tendre après 1895. Cette évolution résultait d'une réorientation de la production vers le bois d'œuvre et donc moins de combustible pour la consommation locale. Alors que l'accroissement naturel des forêts galiciennes fournissait en 1890 davantage de bois de chauffage ( $57 \%$ du total) que de bois d'œuvre ( $43 \%$ ), le ratio s'inversa ensuite, avec $48 \%$ contre $52 \%$ en $1900^{(65)}$. Quant au charbon, il n'était pas utilisable par tous, car nombre de paysans pauvres ne possédaient pas de poêle adapté ${ }^{(66)}$.

61 Calculé d'après les données fournies dans: L. Biegeleisen, Stan ekonomiczny Małopolski (note 34), p. 390-391 et Alexander ENGEL, Österreichs Holz-Industrie und Holzhandel, vol. 2, Vienne, Frick, 1907, p. 391; pour la conversion des tonnes en $\mathrm{m}^{3}$ on a utilisé les équivalences données par M. ENDRES, Handbuch (note 14), p. 581 et supposé que la majorité des bois exportés étaient résineux. Le décalage entre le volume de l'accroissement naturel et de la récolte s'explique par le fait que de nombreuses forêts, en montagne et en Galicie orientale notamment, étaient peu exploitées.

62 «[Ein] Blick aus dem Coupéfenster liefert den Beweis, daß dort, wo in den ersten Jahren nach Eröffnung des Betriebes noch knapp an der Eisenbahn alte Waldungen bestanden haben, nunmehr kahle Berge mit spärlichem Graswuchs [...] dem Reisenden entgegenschauen. Unwillkürlich drängt sich dem Eisenbahner die Frage auf: Was weiter? Wenn wir das Holz und das Petroleum hinausgeführt haben, - und mit dem Holze scheint es bald zur Neige zu gehen - was werden wir weiter "hinaus"führen?...» Ladislaus KrZANOwski, «Eine neue Waldbahn und die Frage des galizischen Holzhandels ", Oesterreichische Eisenbahn-Zeitung, 17 (1894), p. 169-173, ici p. 169.

63 S. G. Bunker, Underdeveloping the Amazon (note 49), p. 223.

64 Cf. par exemple Włodzimierz KAwECKI, Lasy Żywiecczyzny (Les forêts de la région de Żywiec), Żywiec, Towarzystwo Naukowe Żywieckie, 2014, p. 169-170.

65 A. Engel, Österreichs Holz-Industrie und Holzhandel (note 61), p. 391.

66 Przyjaciel Ludu, 29.09.1907. 
Fig. 4: Évolution des prix des combustibles à Cracovie (1890-1914) ${ }^{(67)}$

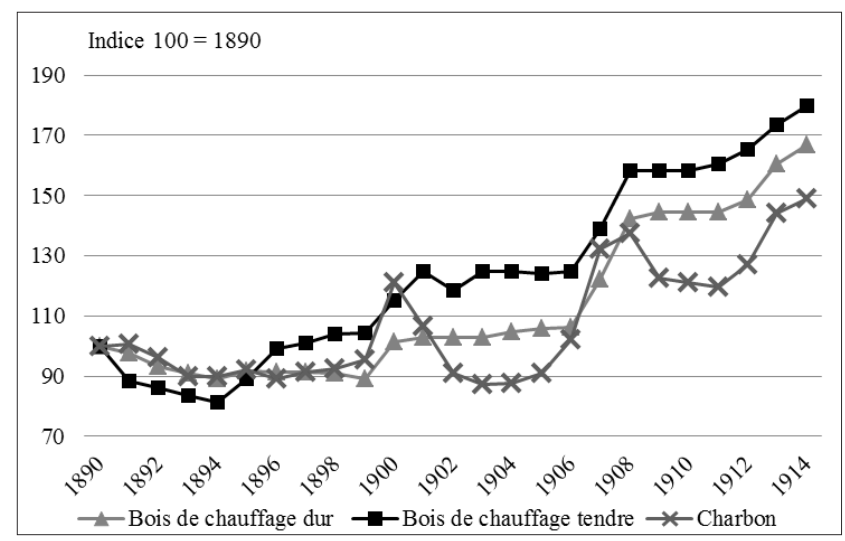

Touchée de plein fouet par la disette des bois, la population ne trouvait plus de combustible à un prix raisonnable. Le poème «L’Avent», publié en 1910 par Wawrzyniec Dąbrowski, résumait la situation:

«L'avent est arrivé. Un vrai problème hante les seuils des pauvres chaumières, parce que l'hiver ne plaisante pas, et que le combustible est cher. Quelle peine pour les forêts, dont le feuillage murmurait des chants à nos ancêtres, aujourd'hui abattues, dont il ne reste que les troncs et les taillis, et donc une pénurie de bois - c'est notre faute, car nous avons vendu les forêts aux Allemands. "Le Polonais est plus malin après coup." L'est-il plus depuis lors?» ${ }^{(68)}$

Ce court poème illustre un phénomène identifié par Bunker à propos de l'Amazonie, à savoir la vulnérabilité des habitants de la périphérie aux changements décidés loin de chez eux ${ }^{(69)}$.

Cette situation était d'autant plus grave qu'en Galicie, la pauvreté et le mauvais état de santé de la population étaient endémiques ${ }^{(70)}$. Comptant parmi les régions les plus pauvres d'Europe, la province était associée à une image d'arriération, comme en atteste l'expression proverbiale de «misère galicienne» popularisée par un célèbre ouvrage de $1888^{(71)}$. À cause de la malnutrition et d'une fréquence élevée de maladies (choléra, typhus, variole, etc.), la Galicie comptait un grand nombre d'hommes inaptes au service militaire ${ }^{(72)}$. Mal

67 Marian Górkiewicz, Ceny w Krakowie w latach, 1796-1914 (Les prix á Cracovie de 1796 á 1914), Poznań, Nakladem Poznanskiego Towarzystwa Przyjaciol Nauk, 1950.

68 «Nadszedł adwent. Szczery kłopot chat nawiedza nieskie progi, zima bowiem nie żartuje, a tak bardzo opał drogi. Szkoda borów, które przodkom swą liściastą pieśń szumiały, dziś wycięte, tylko pieńki, zagajniki z nich zostały, więc brak drzewa - nasza wina, sprzedaliśmy Niemcom bory. "Mądry Polak już po szkodzie.” Czy mądrzejszy od tej pory?» Dodatek Ilustrowany, 51 (1911).

69 S. G. Bunker, Underdeveloping the Amazon (note 49), p. 41.

70 Avec une densité de 102 habitants $/ \mathrm{km}^{2}$. Cf. Mieczyslaw Orlowicz et Kordys Roman, Illustrierter Führer durch Galizien, Vienne/Leipzig, Hartleben, 1914, p. I.

71 Stanisław Szczepanowski, Nędza Galicyi (Misère de la Galicie), Lwów, Gubrynowicz \& Schmidt, 1888; cf. également Krzysztof Zamorski, «La misère de Galicie. Sens et non-sens d'une métaphore historique», in: Jacques Le Rider et Heinz Raschel (dir.), La Galicie au temps des Habsbourg (17721918), Tours, Presses Universitaires François-Rabelais, 2010, p. 315-332.

72 Keely Stauter-Halsted, The Nation In The Village: The Genesis of Peasant National Identity in Austrian Poland, 1848-1914, Ithaca, Cornell University Press, 2005, p. 26. 
nourris, les paysans galiciens travaillaient dans un état «léthargique» et ne parvenaient pas à améliorer leur niveau de vie car ils travaillaient trop peu ${ }^{(73)}$. On conçoit bien que, dans ces conditions, l'enchérissement du bois de chauffage n'ait fait qu'aggraver une situation sociale déjà tendue.

\section{Exploitation forestière, dépendance et sous-développement}

Pour survivre, la population a dû s'adapter. Dans l'arrondissement de Dąbrowski, l'exportation de bois par le Dunajec et la Vistule avait entraîné une envolée des prix et avec elle l'augmentation des vols dans les forêts ${ }^{(74)}$. D'autres témoignages relatent la disparition d'objets en bois de toutes sortes, jusqu'aux panneaux installés sur les puits de pétrole ${ }^{(75)}$. Une autre réponse à la pénurie était l'émigration ${ }^{(76)}$. Dans la plaine sablonneuse et presque complètement déboisée qui s'étendait entre la Wisłoka et le Dunajec, les paysans devaient parcourir jusqu'à 40 kilomètres pour acheter du bois; avec le déboisement, ils avaient aussi perdu les revenus que leur procurait le travail sur les coupes et dans le transport des bois; ils émigrèrent donc en masse vers la Russie et l'Amérique ${ }^{(77)}$. Les départs, qui de façon plus générale auraient durant les années 19111914 concerné jusqu'à $25 \%$ de la population galicienne ${ }^{(78)}$, étaient symptomatiques d'une spirale du sous-développement que l'économie extractive contribuait à alimenter ${ }^{(79)}$. L'intendant forestier du prince Czartoryski, Jan Ligman, l'avait bien compris :

«Nous ne produisons pas de bois pour nos propres besoins, mais pour l'étranger, car il n'y a rien à en faire dans le pays; nous devons nous conformer à ce dont ils ont besoin là-bas. [...] Les forêts ne peuvent fournir du bois à des prix élevés et donner des revenus significatifs que là où la plus grande partie de leurs produits sont utiles au pays, là où l'industrie et les usines fleurissent dans les villes et les bourgs, non pas là où le paysan vit dans une fragile et misérable cabane, le plus souvent avec son bétail» ${ }^{\left({ }^{(0)}\right.}$.

73 Ibid.

74 Przyjaciel Ludu, 29.09.1907.

75 Alison FrAnk, «Environmental, Economic, and Moral Dimensions of Sustainability in the Petroleum Industry in Austrian Galicia», Modern Intellectual History, 8 (2011), p. 171-191, ici p. 179-180.

76 Pour une typologie des stratégies adoptées face à l'insuffisance des ressources forestières, cf. Uwe Schмidt, Der Wald in Deutschland im 18. und 19. Jahrhundert: das Problem der Ressourcenknappheit dargestellt am Beispiel der Waldressourcenknappheit in Deutschland im 18. und 19. Jahrhundert, St. Ingbert, Conte-Verlag, 2002, p. 315.

77 Emil HoŁowkiewicz, «Obrazy fizyograficzne Galicyi (Tableau physique de la Galicie)», Sylwan, 4 (1886), p. 65-70.

78 Norman Davies, Heart of Europe. The Past in Poland's Present, Oxford, Oxford University Press, 2001, p. 331 .

79 Sur ce sujet, cf. également Raphael MAHLER, «The Economic Background of the Jewish Migration from Galicia to the United States", YIVO Annual of Jewish Social Science, 7 (1952), p. 255-267.

80 «My produkujemy drzewo nie na własną potrzebę, ale dla zagranicy, bo go w kraju nie ma na co zużyć; musimy się stosować do tego co tam potrzebują. [...] Tam tylko lasy mogą mieć ceny drzewa wysokie i znaczne dawać dochody, gdzie ich produkta, w największej części w kraju spotrzebowane bywają, gdzie w miastach i miasteczkach przemysł i fabryki kwitną, a włościanin w lichej lub kurnej chacie nie mieszka częstokroć wraz z bydłem.» "Sprawozdanie stenograficzne z obrad VI. Walnego Zgromadzenia Towarzystwa leśnego galicyjskiego w Kołomyji (Compte-rendu sténographique des débats de la VI. Assemblée générale de la Société de forestière galiciennne à Kolomyia)", Sylwan, 6 (1888), p. 353-368, ici p. 354-355. 
La dépendance économique et son corollaire, le retard de développement, constituent depuis l'après-guerre un thème important de l'historiographie polonaise ${ }^{(81)}$. Dans les années 1960-1970, marquées par les dernières décolonisations et les débats sur le néocolonialisme occidental dans les pays du Tiers-monde, Marian Małowist affirmait que le sous-développement moderne était apparu en Europe centrale et orientale, avant même la colonisation de l'Amérique du Sud et des Caraïbes, lorsque les économies de la zone baltique s'étaient à partir de la fin du Moyen Âge spécialisées dans l'exportation de produits agricoles et forestiers ${ }^{(82)}$. Ses écrits furent beaucoup lus en Amérique du Sud, où des penseurs comme Raúl Prebisch développaient la théorie de la dépendance, selon laquelle la pauvreté et l'instabilité des pays du Sud sont les conséquences de processus mis en place par les pays du Nord ${ }^{(83)}$.

Un moyen d'apprécier la dépendance de la Galicie est de comparer sa situation avec d'autres pays exportateurs de bois. Soucieuse de garantir des livraisons pour la consommation locale, la Norvège avait fait interdire en 1892 les exportations depuis le nord du pays afin d'empêcher les marchands étrangers d'y piller les forêts ${ }^{(84)}$. Quant à la Suède, qui disposait de sa propre «colonie interne» dans le Norrland ${ }^{\left({ }^{(85)} \text {, }\right.}$ elle n'avait exporté vers l'Allemagne que 500 wagons de bois bruts contre 52000 wagons de bois sciés en $1902^{(86)}$. Par contraste, la Galicie resta durant toute la période d'étude exportatrice nette de bois de chauffage ${ }^{(87)}$, alors même que la population rurale avait grandement besoin de combustible. Au moment où les exportations vers l'Allemagne étaient au plus fort, des débats sur le sujet éclatèrent à la Diète ${ }^{(88)}$. En 1901, une vingtaine de députés du PSL appelèrent le gouvernement autrichien à prendre des mesures pour limiter l'impact environnemental et économique du déboisement. Dénonçant la mainmise des juifs et des «exploiteurs prussiens» sur le commerce, ils déploraient l'envolée des prix intérieurs et réclamaient une forte taxe à l'export ${ }^{(89)}$. Les initiatives de ce type se multiplièrent durant les années suivantes mais ne débouchèrent sur aucune décision forte.

Rappelant que les tarifs douaniers appliqués par l'Allemagne étaient les mêmes pour la Suède, Paygert s'interrogeait sur les raisons pour lesquelles la Galicie, elle, ne

81 Anna Sosnowska, « Models of East European Backwarness in Post-1945 Polish Historiography», East Central Europe, 32 (2005), p. 125-145.

82 Marian MaŁowist, Wschód a Zachód Europy w XIII-XVI wieku (L'Est et l'Ouest de l'Europe aux XIII'$X V I^{e}$ siècles), Varsovie, Państwowe Wydawnictwo Naukowe, 1973.

83 «Sobre la historia y los historiadores habla Marian Małowist», Estudios Latinoamericanos, 12 (1989), p. 11-39; Eugeniusz Górski, Dependencia y originalidad de la filosofía en Latinoamérica y en la Europa del Este, México, Universidad Nacional Autónoma de México, 1994, p. 9; Raúl Prebisch, Latin America: A Problem in Development, Austin, University of Texas, 1971.

84 C. Lotz, «Expanding the Space» (note 12), p. 269.

85 Ebba Lisberg-Jansen, «La colonie interne du Norrland suédois: modèle d'une périphérie extractive?", L'Espace Politique (en ligne), 2 (2007), https://espacepolitique.revues.org/741 (consulté le 11/02/2016).

86 K. Paygert, «Podstawy» (note 32), p. 477.

87 L. PĄCZEWSKi, Lasy, przemyst $i$ handel drzewny (note 46), p. 163.

88 Przyjaciel Ludu, 01.03.1899, 09.06.1902 et 02.08.1903.

89 Stenographische Protokolle über die Sitzungen des Herrenhauses des Reichsrates, Vienne, Hof -und Staatsdruckerei, 1901, p. 308. 
parvenait pas à surmonter ces difficultés ${ }^{(90)}$. L'une des réponses à cette question réside dans le rôle des élites et la convergence de leurs intérêts avec ceux des marchands allemands. Lorsque la Direction des chemins de fer envisagea, en 1900, d'augmenter le tarif du fret vers l'Allemagne à $10 \%$ de la valeur, la Direction de Goldstein \& Frères s'empressa d'écrire au comte Tarnowski, qui était député à la Diète:

«[Nous] nous autorisons [...] à adresser à M. le comte l'humble requête, dans votre propre intérêt, car cette affaire est aussi de la plus haute importance pour l'exploitation des forêts par les propriétaires galiciens, d'effectuer des démarches en ce sens auprès de la Direction [...] des chemins de fer, et de mettre, dans la mesure du possible, toute votre influence personnelle dans la balance, afin d'éviter [...] l'adoption de cette mesure dommageable au commerce de bois tout entier ${ }^{(91)}$.

Ce courrier confirme l'analyse de Wallerstein, selon laquelle le pouvoir est dans la périphérie exercé par une élite attachée aux relations culturelles et économiques étroites qu'elle possède avec le noyau et par conséquent peu intéressée à libérer la nation ou la région de sa dépendance politique et économique ${ }^{(92)}$. De fait, la grande aristocratie terrienne, qui possédait la plus grande partie des forêts et dominait la Diète de Galicie, fit systématiquement échec aux projets d'interdiction des exportations, auxquels ne s'intéressa pas non plus le pouvoir central à Vienne ${ }^{(93)}$. En 1914, les membres du PSL-Piast, fondé à la suite d'une scission au sein du PSL, présentèrent à nouveau une motion à ce sujet, ce qui montre que le problème n'était pas réglée ${ }^{(94)}$.

La société civile galicienne fut peu active dans ce combat. Dans un article publié en 1911 dans la Gazette de Toruń, Woyczyński soulignait le contraste entre l'engagement des Polonais de Posnanie contre la politique de la Commission de colonisation ${ }^{(95)}$ et l'inaction qui régnait face aux progrès prussiens en Galicie ${ }^{(96)}$. Il en appelait à une mobilisation des chambres de commerce et d'industrie pour aider les propriétaires à s'équiper en scieries ${ }^{(97)}$. Mais peu de mesures furent prises en ce sens. En 1908, le

90 K.PAygert, «Podstawy» (note 32), p. 477.

91 «[Wir] gestatten [...] uns [...] an Ew. Hochgeboren die ergebene Bitte zu richten, im eigenen Interesse, da diese Angelegenheit auch von einschneidenster Bedeutung für die Verwertung des galizischen Waldbesitzers ist, [...] bei den dortigen [...] Staatsbahn-Direktionen in diesem Sinne [...] vorstellig zu werden und so weit als möglich Ihren persönlichen Einfluß zur Vermeidung [...] der den ganzen Holzhandel [...] schädigenden Maßregel in die Wagschale zu werfen. » ANwK 639 n² 2331, p. 237.

92 I. Wallerstein, Capitalist Agriculture (note 41), p. 67-129.

93 Sur la question des intérêts convergents entre les élites galiciennes et autrichiennes, cf. Krzysztof Dunin-WĄsowICZ, "Die sozialen und politischen Bewegungen der polnischen Bauern in Galizien am Ende des 19. und zu Beginn des 20. Jahrhunderts», in: Karlheinz Mack (dir.), Galizien um die Jahrhundertwende: politische, soziale und kulturelle Verbindungen mit Österreich, Vienne/Munich, Verlag für Geschichte und Politik; R. Oldenbourg, 1990, p. 55-67, ici p. 57.

94 Piast, 08.03.1914.

95 Créée en 1886, cette commission devait racheter les propriétés polonaises et y installer des colons allemands. Son action déboucha sur une intense spéculation dont les Polonais, organisés autour d'institutions performantes de crédit, sortirent finalement vainqueurs. Cf. Scott M. EdDIE, «EthnoNationality and Property Rights in Land in Prussian Poland, 1886-1918, Buying the Land from Under the Poles' Feet?», in: Stanley L Engerman et Jacob Metzer (dir.), Land Rights, Ethno-Nationality, and Sovereignty in History, Londres/New York, Routledge, 2004, p. 55-86.

96 Gazeta Toruńska, 23.07.1911.

97 R. Woyczyński, «Przemysł drzewny» (note 38). 
représentant de la Ligue industrielle de Żółkiew déclarait son impuissance face aux dégâts causés par la «hache prussienne»: incapable de peser sur la question du fret, la seule décision qu'il pouvait soutenir était la création d'une grande banque sylvicole ${ }^{(98)}$. Mais ces projets venaient bien tard et ne débouchèrent pas sur des initiatives concrètes. En raison de sa dépendance économique et politique, la Galicie se révéla finalement incapable de résister à la pression du capital allemand sur ses forêts.

\section{Conclusion}

Dans un ouvrage de 1983 dédié à l'histoire économique de la Pologne et à son retard historique de développement, Witold Kula montrait comment les premières nations capitalistes avaient à l'époque moderne installé «leurs plantations de sucre et de coton de l'autre côté de l'Atlantique et leurs "plantations" de grains en Europe orientale» ${ }^{(99)}$. En observant le commerce de bois de la Galicie durant les années 1890-1914, on peut être tenté, par analogie, de désigner ce territoire comme une «colonie du bois» de l'Empire allemand, à l'image des colonies du sucre ou du coton que les Européens avaient massivement établies dans les Caraïbes et sur le continent américain au XVIII ${ }^{\mathrm{e}}$ siècle. En dépit de différences essentielles, comme le recours à l'esclavage ou au travail forcé ${ }^{(100)}$, l'économie forestière galicienne des années 1900 partageait avec les économies de plantation plusieurs de leurs caractéristiques: un haut degré d'intégration au marché mondial depuis la mise en service de nouvelles voies ferrées; l'extraversion, car son développement était largement conduit sur la base d'incitations ou de décisions venues de l'Empire allemand; la dépendance financière, commerciale et technologique visà-vis des capitaux allemands qui dominaient l'activité d'extraction des bois à grande échelle; enfin l'inarticulation entre les différents secteurs de cette activité, car l'exploitation capitaliste des forêts coexistait avec des formes archaïques de récolte des bois pratiquées par la paysannerie locale ${ }^{(101)}$. Les approvisionnements en bois depuis cette région étaient d'autant mieux garantis que les marchands allemands, dont la présence restait discrète et s'appuyait sur tout un réseau d'intermédiaires, ne furent pas confrontés aux formes de résistance, voire de rébellion, auxquelles l'administration forestière prussienne, de son côté, devait faire face dans les provinces polonophones de Prusse et en Afrique orientale ${ }^{(102)}$. En 1897, le Meyers Konversations-Lexikon décrivait la Posnanie comme «la colonie de peuplement qui fait défaut à l'Allemagne dans le monde» ${ }^{(103)}$.

98 Pogoń, 12.01.1908.

99 Witold Kula, Historia, zacofanie, rozwój (Histoire, retard, développement), Varsovie, Czytelnik, 1983, p. 165.

100 Le travail forcé sera cependant utilisé au moment de l'occupation allemande de la forêt de la Białowieża (1915-1918). Cf. T. SunSERI, «Exploiting the Urwald» (note 21), p. 319-322.

101 On reprend ici les éléments de définition de Christian Schnakenbourg, "Contribution à une réflexion théorique sur l'histoire de l'économie de plantation aux Antilles", Cahiers analyse-épistémologie-histoire, 14-15 (1978), p. 167-188.

102 Thaddeus Sunseri, «Reinterpreting a Colonial Rebellion: Forestry and Social Control in German East Africa, 1874-1915», Environmental History, 8 (2003), p. 430-451; Jeffrey K. Wilson, «Environmental Chauvinism in the Prussian East: Forestry as a Civilizing Mission on the Ethnic Frontier, 1871-1914», Central European History, 41 (2008), p. 27-70.

103 Meyers Konversations-Lexikon, vol. 1, Leipzig/Vienne, Bibliographisches Institut, 1897, p. 252. 
De toute évidence, sa principale «colonie du bois» n'était ni le Cameroun, ni la Prusse occidentale, mais bien la Galicie.

\section{Résumé}

L'article interroge la question de l'impérialisme économique dans l'Allemagne wilhelmienne à travers l'exemple du commerce du bois avec la Galicie autrichienne. Après 1890, le chancelier Caprivi initia une politique commerciale offensive dans la Mitteleuropa en concluant des traités commerciaux bilatéraux visant à la réduction des tarifs douaniers. L'une des conséquences de cette politique fut la pénétration commerciale brutale des forêts galiciennes par des compagnies capitalistes d'exploitation du bois basées en Allemagne. En analysant le bilan commercial de la Galicie pour le bois et les produits qui en sont issus, l'article décrit la dépendance croissante de l'économie forestière et de l'industrie $d u$ bois galiciennes vis-à-vis de l'Empire allemand ainsi que l'impact catastrophique de cette évolution sur les populations locales et l'environnement. Il souligne également les similitudes entre les procédés d'extraction de bois appliqués par les firmes allemandes en Galicie et d'autres formes d'exploitation prédatrice des ressources mises en place dans les colonies européennes outre-mer. L'article se demande finalement dans quelle mesure la Galicie peut être considérée comme une «colonie du bois» de l'Empire allemand.

\section{Zusammenfassung}

Der Artikel behandelt den deutschen Holzhandel mit der österreichischen Provinz Galizien und fragt anhand dieses Beispiels nach dem Wirtschaftsimperialismus des Wilhelminischen Kaiserreichs. Ab 1890 förderte der Reichskanzler Caprivi eine offensive Handelspolitik in Mitteleuropa und schloss bilaterale Handelsverträge zur Senkung der Zolltarife. Seine Politik hatte unter Anderem eine brutale kommerzielle Durchdringung kapitalistischer deutscher Holzfirmen in den galizischen Wäldern zur Folge. Anhand der Handelsbilanz Galiziens für Holz und Holzprodukte beschreibt der Artikel die zunehmende Abhängigkeit der galizischen Waldwirtschaft und Holzindustrie gegenüber dem Kaiserreich, sowie die verheerenden Auswirkungen dieser Entwicklung auf die lokale Bevölkerung und auf die Umwelt. Hervorgehoben werden ferner die Gemeinsamkeiten zwischen der von deutschen Firmen in Galizien geführten Holzerzeugung einerseits und Beispielen der europäischen Ressourcenausbeutung in Übersee-Kolonien andererseits. Letztendlich geht der Artikel der Frage nach, inwiefern Galizien als eine deutsche „Holzkolonie“ betrachtet werden kann.

\section{Summary}

The article deals with the German economic imperialism during the Wilhelmine Period, based on the example of timber trade with Austrian Galicia. After 1890, Chancellor Caprivi promoted an offensive commercial policy in Mitteleuropa and concluded numerous bilateral treaties for reduction of tariff barriers. One of the consequences of this policy was the brutal economic penetration of German capitalist logging companies in the Galician forests. By analysing Galicia's trade balance for timber and timber products, the article describes the growing dependency of this region towards the German Empire and the disastrous impact of this process on the local population and environment. It also underlines the similarities between timber extraction led by 
German companies in Galicia and other forms of predatory resource exploitation in the European colonies overseas. Finally, the article discusses in which extent Galicia could be considered as a German «timber colony». 